\section{A 9-year audit of the efficacy of diathermy for cystitis cystica}

Zhuoran Chen, ${ }^{1}$ Lucy Bates, ${ }^{2}$

Nevine Te West, ${ }^{1}$ Kate H. Moore ${ }^{1}$

${ }^{1}$ Department of Urogynaecology, St. George Hospital, University of New South Wales, Sydney; ' ${ }^{2}$ epartment of Urogynaecology, Westmead Hospital, Sydney, Australia

\begin{abstract}
The aim of this study was to assess whether cystoscopy with diathermy for cystitis cystica in patients with recurrent urinary tract infection (UTI) is associated with decreased incidence of UTI._A retrospective 9 -year audit was performed in a single urogynaecology centre. Patients with proven recurrent UTI and failed conservative therapy underwent cystoscopy with diathermy to cystitis cystica. The number of UTI's 12months pre and post cystoscopy was evaluated using women as their own controls with multivariant analysis of cofounding factors. Of 82 patients with recurrent UTI, 47 patients underwent cystoscopy with diathermy to cystitis cystica (median follow up 2 months [interquartile range, IQR6-31, one patient lost to follow up]). Pre-cystoscopy median UTI per annum was 3 per patient (IQR1-4). Post cystoscopy, median UTI for each woman was significantly reduced (median reduction -2[IQR-5-1], mean $-2.14[95 \%$ CI $-2.94, \quad-1.34]$, $\mathrm{P}=<0.001$ ). In the subgroup of patients who had previous vaginal mesh repair for prolapse $(n=8)$ there was no significant benefit from diathermy (median reduction $1.5[95 \%$ CI $-0.988,3.988], \mathrm{P}=0.197)$. In conclusion, cystoscopy and diathermy of cystitis cystica was generally associated with significant reductions in UTI's in the 12 months following diathermy.
\end{abstract}

\section{Introduction}

Cystoscopy is routinely performed to investigate recurrent urinary tract infections (UTI). ${ }^{1}$ If follicular cystitis or cystitis cystica is found at the time of cystoscopy, diathermy to these areas has been performed by Urogynaecologists and Urologists for many years in an attempt to reduce the number of recurrent infections. Prior to commencing this study, there was no published data on the proven efficacy of this practice. Only one report investigating bladder diathermy from 1977, of a small case series of 27 patients with urethral syndrome (frequency/urgency without proven bacterial cystitis), showed that diathermy was more effective than cystoscopy alone. ${ }^{2}$ Therefore, an audit was performed of all women in a tertiary Urogynaecology unit undergoing cystoscopy and diathermy to cystitis cystica or follicular cystitis over 9 years with a primary complaint of recurrent UTI, to determine whether diathermy was associated with a reduced number of infections in the following 12 months.

\section{Materials and Methods}

This was a retrospective audit of patients undergoing diathermy to cystitis cystica or follicular cystitis over a 9-year period. Patients referred to a tertiary Urogynaecology department with recurrent bacterial cystitis, who also had incontinence, prolapse or post coital UTI, were selected for further analysis (patients with a complaint of recurrent UTI alone are not seen in this department and are referred to an Urologist). Recurrent UTI was defined as 3 or more UTI in a 12 -month period. ${ }^{3}$ Patient demographics such as age, menopause status, previous prolapse or incontinence surgery, use of oestriol cream, antibiotics, cranberry, D-Mannose, voiding dysfunction and findings at cystoscopy were recorded (Table 1). Voiding dysfunction was defined as; a max flow rate of $<15 \mathrm{~mL} / \mathrm{sec}$ and/or less that the $10^{\text {th }}$ centile for a voided volume of $200 \mathrm{~mL}$ with a residual $>100 \mathrm{~mL}$. Renal tract ultrasound was performed in these patients to exclude upper renal tract lesions (if positive then patients were referred to urologist) and post void residuals were measured (if $>100 \mathrm{ml}$ patients were taught to double empty). Patients who did not respond to simple measures (correct fluid intake, topical oestriol cream, careful perineal hygiene, careful bladder emptying) [termed non responders] then underwent cystoscopy, sometimes at the same time as concomitant prolapse or stress incontinence surgery. All patients that underwent cystoscopy over a 9-year period were reviewed. The patients who underwent diathermy to areas of cystitis cystica or follicular cystitis (Figure 1) at the time of cystoscopy were then further analyzed. The number of UTI in the 12 months preceding cystoscopy was compared to the number of UTI in the 12 months following cystoscopy and diathermy. Thus, the women were used as their own controls. All women who underwent diathermy were discharged home on a 5-day course of antibiotics. Long-term antibiotics were discussed at their 6-week
Correspondence: Zhuoran Chen, Department of Urogynaecology, St. George Hospital, Cnr Belgrave \& South St, Kogarah, NSW 2217, Australia.

Tel.: +61.02.9113.2272 - Fax: +61.02.9113.3546. E-mail: zhuoranchen2010@gmail.com

Key words: Recurrent urinary tract infection; Cystoscopy; Bladder diathermy; Prolapse surgery.

Contributions: ZC: data collection, analysis, and writing/editing of manuscript; LB: data collection, analysis; NTW: concept design, data collection, analysis; KM: study design, manuscript editing.

Conflict of interest: the authors declare no potential conflict of interest.

Funding: none.

Conference presentation: preliminary data for this study was presented at the International Continence Society meeting in Tokyo, Japan, 2016.

Received for publication: 3 August 2018. Accepted for publication: 21 March 2019.

This work is licensed under a Creative Commons Attribution NonCommercial 4.0 License (CC BY-NC 4.0).

(C) Copyright Z. Chen et al., 2019

Licensee PAGEPress, Italy

Urogynaecologia 2019; 31:223

doi:10.4081/uij.2019.223

postoperative visit based on visual and histopathologic findings.

\section{Statistical analysis}

Baseline data were summarized as median with interquartile range (IQR), as the data were not normally distributed. At the start of this audit, only one published report was available regarding diathermy in 27 patients, but recurrent UTI was not the primary complaint within that study. Therefore, we had no pilot data upon which to base a sample size and simply conducted an audit over the 9-year period. Changes in pre and post cystoscopy UTIs were analyzed via Wilcoxon signed rank test. In order to adjust for confounding factors, a multivariant analysis was performed to evaluate the association between menopause status, previous prolapse or incontinence surgery, voiding dysfunction and antibiotic use, and outcomes, i.e. UTI reduction. Statistical significance was set at $\mathrm{P}<0.05$ and all statistical analysis was performed using SPSS software version 23.0. 


\section{Results}

A total of 82 women with a primary complaint of recurrent UTI along with other gynaecological dysfunction underwent cystoscopy in a 9-year period. In 29 patients, the findings were normal, or only trabeculations, squamous metaplasia, or bladder hyperaemia was found. In 54 women, cystitis cystica and/or follicular cystitis were observed at cystoscopy. Of these patients, 1 was excluded due to previous pelvic radiation, 6 women did not have diathermy due to 1 being on aspirin, and the other 5 had areas too small to diathermy. Thus, these 7 women were excluded from further analysis, leaving a total of 47 women that had a positive cystoscopy and diathermy for review at 12 months.

Median duration of follow up was 12 months (IQR 6-31). Only one woman was lost to follow up. Follow up was less than 12 months in 8/47 (17\%) patients; 1 was referred to a Urologist, 1 moved away, 4 remained UTI free but lived far away and were told to represent if they had further UTI, which they did not. Their GP was contacted subsequently to ensure they had no further UTI. Two failed to attend their follow up appointment, 1 of which represented 7 years later for other pathology.

The baseline characteristics are described in Table 1: the women were largely menopausal and $85 \%$ of these patients were using vaginal oestriol cream. About one third of women had a cystocele and almost half the patients had voiding dysfunction not requiring self-catheterisation. Prophylactic antibiotic was given to $26 / 47$ $(55.3 \%)$ of the women prior to cystoscopy for a median duration of 12 months (IQR: 7.5-16.5). A further 3 women (6.4\%) were using postcoital prophylactic antibiotics only. In the 12 months preceding cystoscopy, overall there was a median of 3 confirmed UTI (IQR 1-4) per women [mean 3.86 (95\% CI 2.9-4.8)]. After cystoscopy and diathermy, the median number of UTI in the following 12 months was markedly reduced to 1 (IQR 0-3), [mean 1.79 (95\% CI $1.22-2.36)$ ] with $34 \%(16 / 47)$ of patients experiencing no UTIs. When using the women as her own control, there was a significant reduction in the number of UTI post cystoscopy (reduction median -2 [IQR -5-1], mean -2.14 [95\% CI -2.94, -1.34]), $\mathrm{P}=<0.001 \quad$ (Figure 2). Subjective selfreported number of UTI's were also reduced post cystoscopy, with no change in mixed growth or contaminated sample numbers (Table 2). For patients who underwent cystoscopy without diathermy, within the 12 month period pre-procedure, there was a median of 2.5 UTIs (IQR 1-4), mean 3.05 (95\% CI 1.8-4.3). In the 12 months post cystoscopy there was a median of 1 UTI (IQR0- 3), mean 1.82 (95\% CI 0.912.72). Mean reduction in number of UTI was not statistically significant $(-1.227$ [95\%CI $-0.106-2.56], \mathrm{P}=0.069)$ in the group who were not diathermied.

Prophylactic antibiotics (generally nitrofurantoin) post cystoscopy was given in $29 / 47(61.7 \%)$ woman with a median duration of 8 months (IQR 4-12) because their follicular cystitis was more severe and/ or extensive. The 3 women that had preoperative post-coital antibiotics remained on prophylaxis. There was no significant difference in UTI reduction rates in patients who had postoperative prophylactic antibiotic to those that did not $(-2.375[95 \%$ CI $0.8-3.9]$ vs -2.1034 [95\% CI -1.04 -3.16],
$\mathrm{P}=0.953$ ). Multivariant analysis of subgroups with potential confounding factors such as menopause status, prolapse, previous incontinence surgery and voiding dysfunction demonstrated no significant effect. However, when comparing patients who had a vaginal mesh repair for prolapse $(n=8)$ versus no mesh $(n=39)$, a different trend was noted. Cystoscopy and diathermy

Table 1. Baseline characteristics pre-cystoscopy.

\begin{tabular}{ll} 
Patient characteristics & \\
Median age at cystoscopy & $\begin{array}{l}70 \text { years } \\
\text { (IQR } 60.75,77)\end{array}$ \\
Post menopausal & $44 / 47(95.6 \%)$ \\
\hline Vaginal oestriol cream use & $40 / 47(85 \%)$ \\
Voiding dysfunction & $22 / 47(46.8 \%)$ \\
\hline Cystocoele & $16 / 47(34 \%)$ \\
Previous prolapse surgery & \\
(1) Native tissue repair & $18 / 47(38 \%)$ \\
(2) Vaginal mesh repair & $8 / 47(17 \%)$ \\
\hline
\end{tabular}

Type of incontinence

(1) No incontinence

(2) Stress incontinence

$6 / 47(12.8 \%)$

(3) Urge incontinence

$7 / 47(14.9 \%)$

(4) Mixed incontinence

$16 / 47(34 \%)$

$18 / 47(38.3 \%)$

Antibiotic Use

(1) Prophylactic once daily $26 / 47(55.3 \%)$

(2) Postcoital only $3 / 47(6.3 \%)$

Anticholinergics

$24 / 47(51.1 \%)$

Hiprex or high dose cranberry $\quad 11 / 47$ (23.4\%)

IQR, interquartile range.

Table 2. Analysis of all urine samples collected in the 12 months pre-cystoscopy and the 12 months post-cystoscopy.

\begin{tabular}{llll} 
& $\begin{array}{l}\text { No. Pre cystoscopy } \\
(\text { Median; IQR) }\end{array}$ & $\begin{array}{l}\text { No. Post cystoscopy } \\
\text { (Median; IQR) }\end{array}$ & P value \\
UTIs confirmed on culture & $3(1-4)$ & $1(0,3)$ & $<0.001$ \\
Self-reported UTI & $3(1.75-6)$ & $0.5(0-1)$ & 0.029 \\
\hline Mixed growth & $0.00(0-1)$ & $0.00(0-1)$ & 0.732 \\
Contaminated sample & $0.00(0-1)$ & $0.00(0-1)$ & 0.897 \\
\hline Pyuria with no growth & $0.00(0-0)$ & $0.00(0-1)$ & 0.765 \\
\hline
\end{tabular}

UTI, urinary tract infection; IQR, interquartile range.

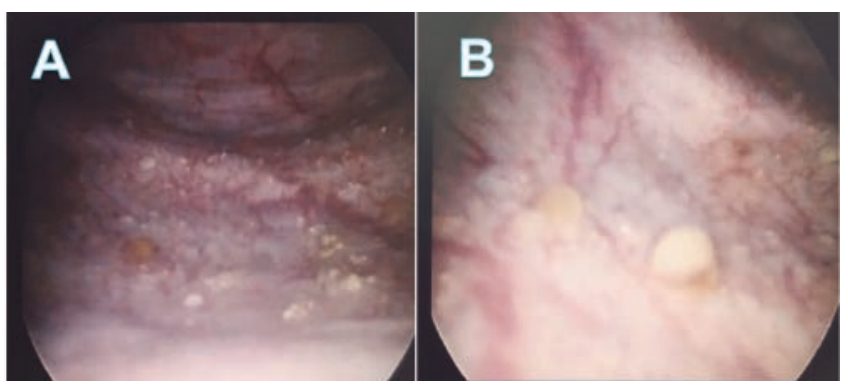

Figure 1. Rigid cystoscopy images demonstrate cystitis cystica (A), with a close up view (B).

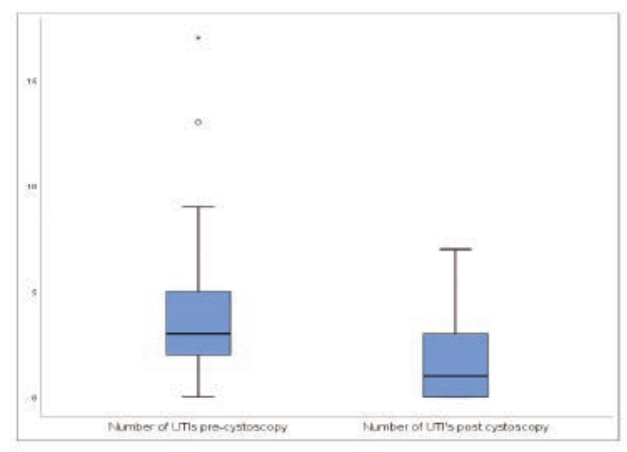

Figure 2. Overall number of urinary tract infections (UTIs) in the 12 months pre- and post- cystoscopy. 
Table 3. Subgroup analysis comparing efficacy of diathermy in patients with or without vaginal mesh.

\begin{tabular}{clll} 
& No. UTI Pre cystoscopy & No. Post cystoscopy & Reduction in No. UTI \\
No mesh (n=39) & & & \\
Mean (SD) & $3.92(3.34)$ & $1.74(1.82)$ & $2.18(95 \%$ CI $1.29,3.07)$ \\
Median (IQR) & $3(1-4)$ & $1(0-3)$ & \\
With mesh (n=8) & & & \\
Mean (SD) & $3.5(2.98)$ & $2.00(2.62)$ & $1.5(95 \%$ CI $-0.99,3.99)$ \\
Median (IQR) & $2.5(1.75-5)$ & $1(0-2.75)$ & \\
\hline
\end{tabular}

UTI, urinary tract infection. IQR, interquartile range.

in the presence of vaginal mesh was not associated with a statistically significant reduction in the number of UTIs postoperatively (Table 3 ).

\section{Discussion}

In this audit we have evaluated 12 months' follow up, after cystoscopy and diathermy for cystitis cystica/ follicular cystitis. This study represented a cohort of 47 women, which was collected in a large unit over 9 years with consistent standard of practice. The audit demonstrates a significant reduction in the number of UTIs, and $34 \%$ UTI-free rate following diathermy.

Recurrent UTIs and its associated antibiotic resistant bacteria are increasingly becoming a global epidemic. ${ }^{4}$ In part intracellular bacterial communities are being recognised as potential reservoirs of recurrent UTI, ${ }^{5-7}$ potentially reflecting a similar pathology to cystitis cystica. Our results support the concept that the cystitis cystica lesions i.e. Figure 1 may act as a reservoir for dormant bacteria which may emerge over time. ${ }^{5}$ Hence diathermy of such lesions may eradicate the bacterial communities and reduce the likelihood of recurrent UTI.

Given that recurrent UTI can often be difficult to manage where there is no anatomical cause for the infection, we were surprised to find at the beginning of our study that there were no recent publications on this topic. Prior to the advent of evidenced based medicine, perhaps clinicians were satisfied that malignancy had been excluded and felt that no further analysis was required. After the commencement of our audit, there has been one report investigating the role of diathermy in women with recurrent UTI, with a short term follow up of 6 months $^{8}$ revealing similar rates of complete resolution of UTIs (32\%) compared to our audit. These authors did not provide information on previous incontinence, prolapse surgery or voiding dysfunction, which could influence baseline rates of UTIs and outcomes. The low rate of vaginal oestriol use in this study $(50 \%)$ as compared $85 \%$ usage in our cohort, may also affect differ- ences in outcomes, especially given evidence for the protective benefits of oestriol against UTI. ${ }^{9}$

In the current era of scrutiny regarding the use of mesh for vaginal prolapse, it was interesting to note that in small sample of women who had recurrent UTI concomitant with previous vaginal mesh history, diathermy did not achieve a similar benefit as those with no history of vaginal mesh. Although numbers are too small to allow any definitive conclusion, future studies in this field should continue to analyse patients with vaginal mesh as a statistical subset.

The present study could be criticized for only reporting 9-year data at a median follow up of 12 months, with 20-year data being preferable. However, we first became aware of the rising incidence of recurrent UTI in women with other urogynaecology problems in 2009 , hence our clinical practice changed in $2011 .{ }^{10}$ This audit was commenced to investigate whether our consistent practice of diathermy resulted in an improved rate of subsequent UTI.

Also, the study could be criticised for the empirical use of postoperative antibiotics in $61.7 \%$ of cases. This clinical practice was guided by level 1 evidence demonstrating efficacy of low dose prophylactic antibiotics ${ }^{11}$ for UTI prevention. Because of the risk of adverse events (e.g. vaginal thrush and gastrointestinal side effects), such therapy was reserved for severe clinical cases. However, it was interesting to find that such long-term postoperative antibiotics conferred no statistical benefit.

\section{Conclusions}

This clinical audit of 9 years of diathermy to cystitis cystica for recurrent UTI has shown a significant reduction the number of UTIs in women in the subsequent 12 months. A randomised controlled trial comparing the effectiveness of diathermy for cystitis cystica versus cystoscopy alone could now be designed, using the present pilot data to ascertain the necessary sample size.

\section{References}

1. Dason S, Dason JT, Kapoor A. Guidelines for the diagnosis and management of recurrent urinary tract infection in women. Can Urol Assoc J 2011;5:316-22.

2. Taylor JS. Diathermy to the trigone and urethera in the management of the female uretheral syndrome. BJU Int 1977;49:4079.

3. Reynard J, Brewster SF, Biers S. Oxford Handbook of Urology. 2nd ed. Oxford: Oxford University Press; 2009. pp 176178.

4. Flores-Mireles AL, Walker JN, Caparon M, Hultgren SJ. Urinary tract infections: epidemiology, mechanisms of infection and treatment options. Nat Rev Microbiol 2015;13:269-84.

5. Rosen DA, Hooton TM, Stamm WE, et al. Detection of intracellular bacterial communities in human urinary tract infection. PLoS Med 2007;4:e329.

6. Cheng Y, Chen Z, Gawthorne JA, et al. Detection of intracellular bacteria in exfoliated urothelial cells from women with urge incontinence. Pathogens Dis 2016;74.

7. Hannan TJ, Totsika M, Mansfield KJ, et al. Host-pathogen checkpoints and population bottlenecks in persistent and intracellular uropathogenic Escherichia coli bladder infection. FEMS Microbiol Rev 2012;36:616-48.

8. Hussain S, Alhalabi F, Zimmern P. Longterm efficacy of fulguration of trigonitis for recurrent urinary tract infections in women. Urol Sci 2015;26: 197-201.

9. Perrotta C, Aznar M, Mejia R, et al. Oestrogens for preventing recurrent urinary tract infection in postmenopausal women. Cochrane Database Syst Rev 2008;2:CD005131

10. Walsh CA, Allen W, Parkin K, et al. Low count bacteriuira in refractory idiopathic detrusor overactivity versus controls. Urogynaecologia 2011;25:11-5.

11. Albert X, Huertas I, Pereiro II, et al. Antibiotics for preventing recurrent urinary tract infection in non-pregnant women. Cochrane Database Syst Rev 2004;2:CD001209. 